\title{
How executive functions are related to intelligence in Williams syndrome
}

\author{
Ana Osório ${ }^{\mathrm{a}, 1}$, Raquel Cruz ${ }^{\mathrm{b}, 1}$, Adriana Sampaio ${ }^{\mathrm{a}}$, Elena Garayzábal ${ }^{\mathrm{c}}$, Rocío Martínez-Regueiro ${ }^{\mathrm{d}}$, \\ Óscar F. Gonçalves ${ }^{\mathrm{a}}$, Ángel Carracedo ${ }^{\mathrm{b}, \mathrm{e}}$, Montse Fernández-Prieto ${ }^{\mathrm{b}, \mathrm{e}, *}$ \\ ${ }^{a}$ Neuropsychophysiology Lab, CIPsi, School of Psychology, University of Minho, 4710-057 Braga, Portugal \\ ${ }^{\mathrm{b}}$ Centro de Investigación Biomédica en Red de Enfermedades Raras (CIBERER) - University of Santiago of Compostela, Spain \\ ${ }^{\mathrm{c}}$ Department of Linguistics, University Autónoma of Madrid, Spain \\ ${ }^{\mathrm{d}}$ Department of Clinical Psychology and Psychobiology, University of Santiago of Compostela, Spain \\ ${ }^{\mathrm{e}}$ Genetic Molecular Unit, Galician Public Foundation of Genomic Medicine, Spain
}

\section{A R T I C L E I N F O}

\section{Article history:}

Received 19 January 2012

Received in revised form 3 February 2012

Accepted 6 February 2012

Available online

\section{Keywords:}

Williams syndrome

Executive functions

Intelligence

\begin{abstract}
A B S T R A C T
Williams syndrome is characterized by impairments in executive functions (EFs). However, it remains unknown how distinct types of EFs relate to intelligence in this syndrome. The present study analyzed performance on working memory, inhibiting and shifting, and its links to IQ in a sample of 17 individuals with WS, and compared them with a group of 17 typically developing individuals matched on chronological age and gender. In conclusion, our results suggest that working memory, inhibiting, and shifting relate differently to intelligence in WS as well as in typical development, with working memory being the EF most closely related to intelligence in both groups. Notably, the magnitude of the associations between the three EFs and IQ was substantially higher in the WS group than in the TD group, bringing further confirmation to the notion that frontal lobe impairments may produce a general compromise of several EFs.
\end{abstract}

(c) 2012 Elsevier Ltd. All rights reserved.

\section{Introduction}

Williams syndrome (WS) is a rare neurodevelopmental disorder caused by a submicroscopic deletion on the long arm of chromosome 7q11.23 (Korenberg et al., 2000), with an approximate incidence of 1 in 7500 live births (Strømme, Bjømstad, \& Ramstad, 2002). This syndrome is characterized by a distinctive pattern of physical (e.g. facial dysmorphia), medical (e.g. cardiovascular problems), socio-emotional (e.g. heightened empathy) and cognitive (e.g. moderate mental delay) features (Bellugi, Korenberg, \& Klima, 2001; Mervis \& Klein-Tasman, 2000; Sampaio et al., 2009). Individuals with WS have been of interest to researchers in the area of cognitive neuroscience because of their distinct cognitive profile of peaks and valleys, with relative verbal strengths contrasting with weaknesses in non-verbal domains (Atkinson et al., 2003; Bellugi, Lichtenberger, Jones, Lai, \& St. George, 2000; Farran, Jarrold, \& Gathercole, 2001; Mervis \& Klein-Tasman, 2000; Semel \& Rosner, 2003). Furthermore, individuals with WS show ample evidence of frontal lobe dysfunction (Menghini, Addona, Costanzo, \& Vicari, 2010; Mobbs et al., 2007; Rhodes, Riby, Park, Fraser, \& Campbell, 2010). In fact, these individuals show a higher prevalence of hyperactive and impulsive symptoms than expected, with attention-deficit/hyperactivity disorder (ADHD) as one of the most frequent co-morbid diagnoses (Dodd \& Porter, 2009; Kennedy, Kaye, \& Sadler, 2006).

\footnotetext{
* Corresponding author at: Fundación Pública Galega de Medicina Xenómica, Complexo Hospitalario Universitario de Santiago, Choupana s/n Edificio Consultas planta-2, 15706 Santiago de Compostela (A Coruña), Spain. Tel.: +34 981 951490; fax: +34 981951473.

E-mail address: montse.f.prieto@gmail.com (M. Fernández-Prieto).

${ }^{1}$ Share equal first authorship.
} 
There has been increasing interest in studying the level of impairment in the executive functioning of individuals with WS. The term "executive function" (EF) refers to a range of processes such as working memory, inhibitory control and attentional shifting, which are believed to depend on frontal lobe activity and underlie goal-directed responses to novel and challenging situations (Friedman et al., 2006; Miyake et al., 2000). Porter, Coltheart, and Langdon (2007) found that a sample of 20 individuals with WS (aged 5-46 years) displayed significantly poorer response inhibition than expected based on their mental age and intellectual functioning. Rhodes et al. (2010) compared the performance of a sample of 19 individuals with WS (11-29 years of age) on a set of three EF tasks with two control groups: a chronological age and gender-matched group and a verbal ability and gender-matched group. They found that the WS group presented with impairments compared to typically developing groups in all three tasks, which involved attention set-shifting, spatial working memory and planning. Menghini et al. (2010) extended these results by providing evidence of deficits in both verbal and visuospatial EF tasks in WS participants compared with mental age-matched controls.

One important issue that has been neglected is how distinct types of executive functions relate to intelligence in WS. Because this syndrome is characterized by frontal lobe dysfunction, which is associated with executive impairments, it seems necessary to study the pattern of associations between different types of EF and intelligence. As no WS studies have been conducted on this subject, we review evidence from normative samples. Although measures of EF are moderately correlated in typical development, they are not redundant, which suggests that that they may have different associations with intelligence (Miyake et al., 2000). Indeed, over the course of the past two decades, research with normative samples has shown that inhibiting, working memory and shifting display distinct patterns of association with general cognitive ability. Regarding intelligence and inhibiting, Anderson and Spellman (1995) state that inhibition is a necessary mechanism in human cognition that relies on both excitatory and inhibitory processes and contributes to the stability of the neural networks. Despite scant empirical supporting evidence, the role of inhibition is accepted as being present in several cognitive skills such as selective attention, language comprehension and production, memory retrieval and analogical reasoning (Anderson \& Spellman, 1995; Das, 2002; Michel \& Anderson, 2009). In addition, Dempster (1991) and Dempster and Corkill (1999) reviewed the available evidence and concluded that inhibition seems to be an important factor in intelligence in nonclinical samples. More recently, Polderman et al. (2009) found an association between inhibitory control and intelligence in 9-, 12-, and 18-year-olds. Regarding working memory, a meta-analysis of 86 samples found evidence of moderate to strong correlations between working memory and intellectual ability (Ackerman, Beier, \& Boyle, 2005). Indeed, working memory has been implicated in distinct mechanisms of human cognitive function (Jarrold \& Towse, 2006). Some studies have failed to confirm a link between intelligence and shifting (Friedman et al., 2006; Rockstroh \& Schweizer, 2001). However, Ardila, Pineda, and Rosselli (2000) reported correlations between the Wisconsin Card Sorting Test, a measure of shifting, and adult IQ on the WAIS. Similar results were found in a sample of children aged 3-6 years, whose performance on a dimensionalchange card sort task (with high demands in terms of shifting) was strongly correlated with measures of intelligence (Hongwanishkul, Happaney, Lee, \& Zelazo, 2005). Interestingly, only one study has analyzed the association between performance on tasks requiring all three types of EF and intelligence (Friedman et al., 2006). The authors found that updating working memory significantly correlated with intelligence in a very large sample of healthy adolescents aged 16-18 years, although the same results were not found with inhibiting and shifting.

To date, there have been no similar studies exploring the possible links between distinct types of EF and intelligence in WS.

The purpose of the present work was threefold. First, we compared performance on tasks assessing working memory, inhibiting and shifting between two groups: (a) a group of individuals with WS aged 10-29 years and (b) a group of typically developing individuals matched by chronological age and gender. Given the wealth of studies suggesting that frontal lobe impairments are a characteristic of this syndrome, and based on prior evidence, we expect individuals with WS to show significantly poorer performance than TD individuals on all three types of EF.

Second, we aimed to explore the pattern of associations between the three types of EF. We expect significant intercorrelations for both groups (for evidence for the TD group, see Friedman et al., 2006; Miyake et al., 2000), but these intercorrelations may be particularly evident in the clinical group. Because WS is characterized by frontal lobe impairments, we anticipate that all measures of EF may be affected and may vary together.

Finally, we aimed to explore the pattern of associations among the three types of EF (working memory, inhibiting and shifting) and intelligence in the two groups. In accordance with a recent report by Friedman et al. (2006), we posit that only working memory is associated with intelligence in the TD group. Due to frontal lobe impairments in the WS group, a more general compromise of EF may be present, which may be reflected in significant associations between EF and intelligence measures.

\section{Materials and methods}

\subsection{Participants}

Two groups of participants took part in this study. Seventeen participants with a diagnosis of WS (10 females and 7 males, with ages ranging from 9.6 to 29.3 years $(M=20.6, \mathrm{SD}=5.9)$ ) with previously confirmed positive fluorescence in situ hybridization (FISH) to an elastin gene deletion in chromosome 7 (Ewart et al., 1993) were recruited at Fundación Pública Galega de Medicina Xenómica (Santiago de Compostela, Spain). The exclusion criteria were the presence of any sensorial or speech disorder, as well as comorbidity with severe psychopathology not associated with the syndrome. The control group 
Table 1

Socio-demographic data for the WS group and the control group.

\begin{tabular}{|c|c|c|c|c|}
\hline & \multicolumn{2}{|c|}{ Williams syndrome $(n=17)$} & \multicolumn{2}{|c|}{ Controls $(n=17)$} \\
\hline & $M(\mathrm{SD})$ & Range & $M(\mathrm{SD})$ & Range \\
\hline Age & $20.6(5.9)$ & $9.6-29.3$ & $19.0(5.7)$ & $10.1-28.5$ \\
\hline Sex & $N$ & $\%$ & $N$ & $\%$ \\
\hline Male & 7 & $41.2 \%$ & 7 & $41.2 \%$ \\
\hline Female & 10 & $58.8 \%$ & 10 & $58.8 \%$ \\
\hline
\end{tabular}

was composed of typically developing individuals without a history of sensorial, psychiatric, or neurological disorder or cognitive impairment. This group was matched with the WS group based on gender and age $(M=19.0, S D=5.7)$. After a complete description of the study, each participant (or their parents) provided written informed consent for their participation in the study. Table 1 displays the socio-demographic characteristics of the sample.

\subsection{Neurocognitive assessment}

\subsubsection{General cognitive functioning}

Wechsler intelligence scales - The WISC-IV (Weschler, 2003) and WAIS-III (Wechsler, 1997) are used to assess general cognitive functioning in individuals 16 and under and 16 and over, respectively. These scales are two of the most frequently used international systems for assessing intellectual quotient (Full scale IQ - FSIQ), in normative and clinical samples, and they have been widely used in the WS literature (e.g. Capitão et al., 2011; Howlin, Davies, \& Udwin, 1998; Searcy et al., 2004; Udwin, Davies, \& Hosylin, 1996).

\subsubsection{Working memory}

Working memory was determined by the raw score on the reverse digit span subtest of the WISC or WAIS. In this specific part of the subtest, the experimenter reads increasingly longer sequences of digits aloud and asks the participant to repeat each sequence in reverse order. This task can be considered a good index of verbal working memory because it involves the temporary storage and manipulation of verbal information.

\subsubsection{Shifting}

Trail making test - TMT (Raitan, 1958) - This test is composed of two conditions: part A involves drawing lines between numbers from 1 to 15 and part $B$ requires the participant to link numbers and letters in an alternate sequence. Part $B$ requires more shifting ability than part A and is clearly the more sensitive part of the test (Spreen \& Strauss, 1991).

Wisconsin card sorting test - WCST (Heaton, 1981) - This task requires participants to match stimulus and response cards according to three alternative criteria (color, quantity or shape). The participant must place each response card under one of the four reference cards according to these criteria. As the test progresses, unexpected shifts in the matching rule require the patient to change his/her strategy. The test assesses "set-shifting", the ability to display flexibility when faced with changing schedules of reinforcement, perseveration and abstract thinking.

\subsubsection{Inhibiting}

The Stroop task (Stroop, 1935) requires the inhibition of a competing response (inhibiting an over-learned response with respect to a more controlled one). This task involves three consecutive conditions. In the first condition (word reading; words printed in black), participants are asked to read as many words as possible in $45 \mathrm{~s}$. The second condition (color naming) consists of naming the color of Xs printed in red, green or blue. In the third condition (incongruent word and color), participants are asked to name the color of the written words, but the words are incongruent (e.g. the word "blue" is written in red).

Go/no-go task - The go/no-go task used in this study was adapted (for details, see Capitão et al. (2011)) from the standard go/no-go paradigm that has been used for decades to test behavioral inhibition (Costantini \& Hoving, 1973). Participants are asked to press a key as rapidly as possible whenever they see the target stimulus (letter A - go cue) and to withhold responses to the non-target stimulus (letter X - no-go cue). Of a total of 100 stimuli, $90(90 \%)$ are go cues and $10(10 \%)$ are no-go cues. The high frequency of go cues creates a response tendency that must be inhibited for no-go cues, providing a measure of the ability to inhibit responses. In each trial, a single letter appears and remains on the center of the screen until the participant answers or $3 \mathrm{~s}$ elapse. The trial order was randomized for each participant. The letters $A$ and $X$ were of the same size and color (black). This task was compiled and run on a laptop computer using Superlab 4.0.7.2.3.3 (Cedrus Corporation, San Pedro, CA, United States).

\subsection{Procedure}

After explaining the goals of the research to the participants, their socio-demographic, diagnostic and clinical information was obtained, along with their consent forms. The tests were administered at the Fundación Pública Galega de Medicina 
Xenómica or in public/private schools. Participants were assessed individually in 3 sessions lasting approximately 2 h each. The order of the tasks was randomized for each participant. All tasks included a practice phase, and the experimenter ensured that participants understood the instructions.

\subsection{Statistical methods}

Statistical calculations were performed using PASW Statistics 18 (IBM SPSS Statistics). ANOVAs were conducted to test for differences in EF performance between individuals with WS and controls. Due to significant correlations between many of the EF tasks and IQ, the latter was included as a covariate to determine the existence of additional (non IQ-related) differences between the clinical and the control groups.

To explore the pattern of relationships between different EF tasks and IQ in both groups, three composite variables were defined: working memory, inhibiting and shifting. Working memory was directly estimated from the reverse digit span. Inhibiting was defined as a combination of interference in the Stroop task and go/no-go performance, and shifting was defined as a function of time difference in the TMT (TMT B-A difference) as well as perseverative responses and perseverative errors in the WCST. Subsequently, correlations between these variables, as well as correlations with the subjects' IQ, were calculated. A multiple regression of the composite variables on IQ was also performed to test the independent effect of each variable.

\section{Results}

The age and gender distribution of the sample stratified by group is shown in Table 1 .

Table 2

The results of the ANOVAs for individual EF tasks.

\begin{tabular}{|c|c|c|c|c|c|c|c|c|}
\hline & \multicolumn{3}{|c|}{ Williams syndrome } & \multicolumn{3}{|c|}{ Controls } & \multicolumn{2}{|l|}{$F$} \\
\hline & $M$ & SD & $n$ & $M$ & SD & $n$ & Without IQ & With IQ \\
\hline \multicolumn{9}{|l|}{ Go/no go } \\
\hline Response time $(i)$ & 606.7 & 246.7 & 11 & 378.3 & 63.3 & 15 & $11.98^{* *}$ & $<0.01$ \\
\hline Correct go & 98.4 & 2.3 & 11 & 100.0 & 0.0 & 15 & $7.21^{*}$ & 0.24 \\
\hline Correct no-go & 76.4 & 23.4 & 11 & 83.3 & 12.3 & 15 & 0.98 & 0.21 \\
\hline False positives $(i)$ & 23.6 & 23.4 & 11 & 16.7 & 12.3 & 15 & 0.98 & 0.21 \\
\hline Omissions $(i)$ & 1.6 & 2.3 & 11 & 0.0 & 0.0 & 15 & $7.21^{*}$ & 0.24 \\
\hline \multicolumn{9}{|l|}{ Stroop } \\
\hline No. correct word reading & 55.9 & 19.1 & 17 & 121.1 & 29.0 & 16 & $58.78^{* * *}$ & 2.41 \\
\hline No. correct color naming & 37.9 & 13.7 & 17 & 77.8 & 13.5 & 16 & $70.97^{* * *}$ & $5.33^{*}$ \\
\hline No. correct word-color naming & 39.4 & 22.6 & 17 & 50.6 & 10.9 & 16 & 3.18 & 1.87 \\
\hline Interference (i) & 13.4 & 30.6 & 17 & -99.1 & 36.2 & 16 & $93.24^{* * *}$ & $33.73^{* * *}$ \\
\hline \multicolumn{9}{|l|}{ TMT } \\
\hline TMT A - errors & 0.9 & 1.7 & 17 & 0.2 & 0.4 & 17 & 3.09 & 0.13 \\
\hline TMT A - average & 24.5 & 4.3 & 17 & 24.5 & 3.9 & 17 & $<0.01$ & 0.62 \\
\hline TMT A - SD & 8.2 & 2.2 & 17 & 8.8 & 1.4 & 17 & 1.02 & 0.78 \\
\hline TMT A - time & 74.4 & 51.2 & 17 & 20.1 & 10.6 & 17 & $18.30^{* * *}$ & $<0.01$ \\
\hline TMT B - errors & 7.3 & 7.3 & 13 & 0.6 & 1.3 & 17 & $13.69^{* * *}$ & 0.10 \\
\hline TMT B - average & 51.6 & 8.3 & 14 & 49.4 & 7.8 & 17 & 0.57 & 0.23 \\
\hline TMT B - SD & 14.7 & 2.4 & 14 & 15.0 & 1.1 & 17 & 0.12 & 0.71 \\
\hline TMT B - time & 165.2 & 124.2 & 14 & 41.2 & 16.6 & 17 & $16.70^{* * *}$ & 0.03 \\
\hline TMT B-A time $(s)$ & 99.8 & 130.3 & 14 & 21.1 & 14.9 & 17 & $6.15^{*}$ & $<0.01$ \\
\hline \multicolumn{9}{|l|}{ WCST } \\
\hline No. non-perseverative errors & 25.7 & 13.2 & 16 & 9.3 & 17.6 & 16 & $8.97^{* *}$ & 0.19 \\
\hline No. perseverative errors $(s)$ & 40.1 & 17.9 & 16 & 15.5 & 24.5 & 16 & $10.55^{* *}$ & 1.51 \\
\hline No. errors - total & 69.3 & 17.3 & 16 & 18.7 & 18.5 & 16 & $64.11^{* * *}$ & 0.45 \\
\hline No. attempts & 125.6 & 6.7 & 16 & 83.3 & 8.8 & 15 & $226.99^{* * *}$ & $16.38^{* * *}$ \\
\hline Conceptual errors & 37.8 & 21.0 & 16 & 64.5 & 7.4 & 15 & $21.61^{* * *}$ & 0.20 \\
\hline No. correct responses & 60.1 & 16.5 & 16 & 69.0 & 7.7 & 15 & 3.61 & 0.46 \\
\hline No. perseverative responses $(s)$ & 53.1 & 27.6 & 16 & 23.4 & 22.6 & 16 & $11.05^{* *}$ & 0.93 \\
\hline \multicolumn{9}{|l|}{ WISC/WAIS } \\
\hline Full scale IQ & 55.2 & 7.8 & 17 & 113.2 & 12.2 & 17 & $273.22^{* * *}$ & - \\
\hline Reverse digit span $(w)$ & 3.2 & 1.6 & 17 & 9.1 & 2.8 & 17 & $57.07^{* * *}$ & 2.19 \\
\hline
\end{tabular}

$i$, variables included in the composite for inhibiting; $s$, variables included in the composite for shifting; $w$, variable for working memory.

Italics: $p<0.0015$ (Bonferroni adjusted $p$ value for 33 independent tests).

$* p<0.05$.

** $p<0.01$.

*** $p<0.001$. 
Table 3

Matrix correlation between composite variables.

\begin{tabular}{|c|c|c|}
\hline & Shifting & Inhibiting \\
\hline \multicolumn{3}{|l|}{ Controls } \\
\hline Working memory & $-0.405(n=16)$ & $-0.296(n=14)$ \\
\hline Shifting & & $-0.053(n=13)$ \\
\hline \multicolumn{3}{|l|}{ Williams syndrome } \\
\hline Working memory & $-0.378(n=13)$ & $-0.859^{* * *}(n=11)$ \\
\hline Shifting & & $0.307(n=8)$ \\
\hline
\end{tabular}

\subsection{Differences in individual EF between WS and TD}

Table 2 presents the descriptive statistics for individual EF tasks for both groups, as well as the results of the ANOVAs and ANCOVAs (adjusting for IQ) between WS participants and controls. Significant differences were found, especially in the Stroop test and the WCST. However, most of these differences disappeared when adjusting for IQ. In contrast, even after controlling for IQ differences in interference in the Stroop test and the number of attempts on the WCST remained significant (considering the Bonferroni correction).

\subsection{Correlations between EF composite variables}

Pearson correlation coefficients between each pair of composite variables are shown in Table 3. The only significant correlation was observed between working memory and inhibiting in the WS group. Correlation coefficients in the TD group were lower and non-significant, despite the larger $N$ of this group. Effect sizes were medium to large in the WS group. For the TD group, medium effect sizes were found between working memory and shifting as well as between working memory and inhibiting, whereas the effect size between shifting and inhibiting was small. Notably, for shifting and inhibiting, higher scores represented worse performances, leading to the presence of negative correlations.

\subsection{Correlations between EF composite variables and IQ}

Pearson correlation coefficients between the composite variables and IQ are shown in Table 4. In the TD group, only working memory was significantly correlated with IQ with $38 \%$ of shared variance. The variance shared between IQ and shifting and inhibiting was $7.7 \%$ and $0.66 \%$, respectively. The effect sizes pertaining to these correlation coefficients were small. For the WS group, working memory and inhibiting were significantly associated with IQ (64\% and $65 \%$ of shared variance, respectively). Shifting and IQ were not significantly correlated (23\% of shared variance), although the effect size was medium to large.

There were two differences in the pattern in both groups. First, the correlation coefficient between working memory and IQ was higher in the WS group. Second, within the clinical group, IQ was also significantly correlated with inhibiting. However, this effect seems to be related to the previously observed correlation between inhibiting and working memory in WS individuals (see Table 3). In fact, when both variables (working memory and inhibiting) were included in the same regression model on IQ working memory was the only variable included in the regression model when a stepwise variable selection procedure was used. Working memory was positive and significantly related to IQ $(b=3.98, p=0.0011)$. This regression model $\left(F_{1,10}=22.46, p=0.0011\right)$ explained $71.4 \%$ of the variance.

\section{Discussion}

Previous studies have found that individuals with WS show impairments in executive functioning (Menghini et al., 2010; Porter et al., 2007; Rhodes et al., 2010). However, to date, no study has examined the pattern of associations between distinct types of EF measures and intelligence in this syndrome. The present study is the first to analyze how working memory, shifting and inhibiting are related to IQ in a sample of individuals with WS. This study also explored the intercorrelations among these three types of EF in WS, thus expanding prior results obtained in normative samples.

Table 4

Pearson correlation coefficients between IQ and composite variables in Williams syndrome and controls.

\begin{tabular}{|c|c|c|c|}
\hline & Working memory & Shifting & Inhibiting \\
\hline Controls & $0.618^{* *}(n=17)$ & $-0.278(n=16)$ & $0.081(n=14)$ \\
\hline Williams syndrome & $0.797^{* * *}(n=17)$ & $-0.479(n=13)$ & $-0.807^{* *}(n=11)$ \\
\hline
\end{tabular}


First, our results confirm previous evidence because the performance of individuals with WS was significantly poorer on almost all measures of EF when compared with TD individuals. Notably, when IQ was controlled for, most of the differences between the two groups lost statistical significance. This finding seems to indicate that general cognitive ability may account for most of the differences between WS and TD in terms of executive functioning. Interestingly, even when controlling for IQ, the WS group showed significantly lower interference in the Stroop task. This seemingly contradictory result may be explained by the reading difficulties that are also characteristic of this syndrome. Therefore, naming a color not denoted by a written word may be easier for these individuals because it requires less inhibiting ability.

Our second aim was to explore the pattern of correlations among the different EFs in both the WS and TD groups. We expected that the frontal lobe impairments in the clinical group (Menghini et al., 2010; Porter et al., 2007; Rhodes et al., 2010) would translate into significant correlations among the different EF (Rabbitt, Lowe, \& Shilling, 2001). Previous evidence supported an expectation of significant intercorrelations for the TD group (Friedman et al., 2006; Miyake et al., 2000). Our results showed that working memory and inhibiting were significantly correlated in the WS group, but not in the TD group. Furthermore, all other correlation coefficients were non-significant for both groups. Because the samples were rather small, an analysis of effect sizes could provide a better picture of the pattern of associations. Indeed, the effect sizes were consistently medium to large in the WS group, suggesting that a larger sample would have allowed us to detect significant correlations among all of the EFs. A similar trend was observed for the TD group: medium effect sizes were found for the correlations between working memory and shifting and between working memory and inhibiting. Once again, these results corroborate prior evidence (Friedman et al., 2006; Miyake et al., 2000) suggesting that distinct EFs are connected, but separable. In contrast, the effect size of the correlation between shifting and inhibiting was small in our sample of TD individuals. This result may also be due to the different tasks selected for the assessment of these EFs in distinct papers.

Our final aim was to explore the pattern of associations among EF and intelligence measures in the two groups. Our data for the TD group confirmed previous results by Friedman et al. (2006) by associating working memory only with intelligence. Indeed, the variance shared between these two variables was quite high, whereas the variance shared between IQ and shifting and inhibiting was almost residual. A similar pattern was found for the WS group. Working memory was significantly associated with IQ (more than half of their variance was shared), and this association was even greater in magnitude than the one observed in the TD group. Additionally, inhibiting was found to correlate significantly with IQ. However, in a stepwise multiple regression, only working memory was selected to be included in the model to predict IQ. Thus, the individual correlation found between inhibiting and IQ was likely due to the aforementioned correlation between inhibiting and working memory in the clinical group. Finally, although shifting and IQ were not significantly correlated, they shared more than one-fifth of their variance.

\section{Conclusions}

Our results suggest that working memory, inhibiting and shifting relate differently to intelligence in WS and in typical development. Despite the small sample size, these results confirm previous evidence on typically developing individuals (Friedman et al., 2006) and expand current knowledge on the relationship between EF and intelligence in WS. In our study, working memory was the EF most closely related to intelligence in both groups. It is notable that the magnitude of the associations between the three EFs and IQ was substantially higher in the WS group than in the TD group. This finding further confirms the notion that frontal lobe impairments may produce a general compromise of several EFs (Rabbitt et al., 2001).

Further investigations in larger samples, with wider age spans and using alternative EF measures are warranted to verify the generalizability of the results reported here.

\section{Acknowledgments}

This research was supported by the Portuguese Foundation for Science and Technology (grant PTDC/PSI-PCL/115316/ 2009) and by Fundación Alicia Koplowitz (5th Call for Grants for Research in Childhood and Adolescent Psychiatry Early Neurodegenerative Diseases).

\section{References}

Ackerman, P. L., Beier, M. E., \& Boyle, M. O. (2005). Working memory and intelligence: The same or different constructs? Psychological Bulletin, 131(1), 30. Anderson, M. C., \& Spellman, B. A. (1995). On the status of inhibitory mechanisms in cognition: Memory retrieval as a model case. Psychological Review, $102(1), 68$. Ardila, A., Pineda, D., \& Rosselli, M. (2000). Correlation between intelligence test scores and executive function measures. Archives of Clinical Neuropsychology, $15(1), 31-36$.

Atkinson, J., Braddick, O., Anker, S., Curran, W., Andrew, R., Wattam-Bell, J., et al. (2003). Neurobiological models of visuospatial cognition in children with Williams syndrome: Measures of dorsal-stream and frontal function. Developmental Neuropsychology, 23(1-2), 139-172.

Bellugi, U., Korenberg, J. R., \& Klima, E. S. (2001). Williams syndrome: An exploration of neurocognitive and genetic features. Clinical Neuroscience Research, 1(3), 217-229.

Bellugi, U., Lichtenberger, L., Jones, W., Lai, Z., \& St. George, M. (2000). The neurocognitive profile of Williams syndrome: A complex pattern of strengths and weaknesses. Journal of Cognitive Neuroscience, 12(Suppl. 1), 7-29.

Capitão, L., Sampaio, A., Férnandez, M., Sousa, N., Pinheiro, A., \& Gonçalves, Ó. F. (2011). Williams syndrome hypersociability: A neuropsychological study of the amygdala and prefrontal cortex hypotheses. Research in Developmental Disabilities, 32, 1169-1179.

Costantini, A., \& Hoving, K. (1973). The effectiveness of reward and punishment contingencies on response inhibition. Journal of Experimental Child Psychology, 16(3), 484-494. 
Das, J. (2002). A better look at intelligence. Current Directions in Psychological Science, 11(1), 28.

Dempster, F. N. (1991). Inhibitory processes: A negleted dimension of intelligence. Intelligence, 15(2), 157-173.

Dempster, F. N., \& Corkill, A. J. (1999). Individual differences in susceptibility to interference and general cognitive ability. Acta Psychologica, 101(2-3), 395-416.

Dodd, H. F., \& Porter, M. A. (2009). Psychopathology in Williams syndrome: The effect of individual differences across the life span. Journal of Mental Health Research in Intellectual Disabilities, 2(2), 89-109.

Ewart, A. K., Morris, C. A., Atkinson, D., Jin, W., Sternes, K., Spallone, P., et al. (1993). Hemizygosity at the elastin locus in a developmental disorder, Williams syndrome. Nature Genetics, 5(1), 11-16. doi:10.1038/ng0993-11.

Farran, E. K., Jarrold, C., \& Gathercole, S. E. (2001). Block design performance in the Williams syndrome phenotype: A problem with mental imagery? Journal of Child Psychology and Psychiatry, 42(6), 719-728.

Friedman, N. P., Miyake, A., Corley, R. P., Young, S. E., DeFries, J. C., \& Hewitt, J. K. (2006). Not all executive functions are related to intelligence. Psychological Science, $17(2), 172$.

Heaton, R. K. (1981). Wisconsin card sorting test, manual. Odessa, FL: Psychological Assessment Resources.

Hongwanishkul, D., Happaney, K. R., Lee, W. S. C., \& Zelazo, P. D. (2005). Assessment of hot and cool executive function in young children: Age-related changes and individual differences. Developmental Neuropsychology, 28(2), 617-644.

Howlin, P., Davies, M., \& Udwin, O. (1998). Cognitive functioning in adults with Williams syndrome. Journal of Child Psychology and Psychiatry, 39(2), 183-189.

Jarrold, C., \& Towse, J. N. (2006). Individual differences in working memory. Neuroscience, 139(1), 39-50.

Kennedy, J. C. M. D. , Kaye, D. L. M. D. , \& Sadler, L. S. M. D. (2006). Psychiatric diagnoses in patients with Williams syndrome and their families. Jefferson Journal of Psychiatry, 20(1), 4.

Korenberg, J. R., Chen, X. N., Hirota, H., Lai, Z., Bellugi, U., Burian, D., et al. (2000). Genome structure and cognitive map of Williams syndrome. Journal of Cognitive Neuroscience, 12(Suppl. 1), 89-107.

Menghini, D., Addona, F., Costanzo, F., \& Vicari, S. (2010). Executive functions in individuals with Williams syndrome. Journal of Intellectual Disability Research, 54(5), 418-432.

Mervis, C. B., \& Klein-Tasman, B. P. (2000). Williams syndrome: Cognition, personality, and adaptive behavior. Mental Retardation and Developmental Disabilities Research Reviews, 6(2), 148-158.

Michel, F., \& Anderson, M. (2009). Using the antisaccade task to investigate the relationship between the development of inhibition and the development of intelligence. Developmental Science, 12(2), 272-288.

Miyake, A., Friedman, N. P., Emerson, M. J., Witzki, A. H., Howerter, A., \& Wager, T. D. (2000). The unity and diversity of executive functions and their contributions to complex. Cognitive Psychology, 41(1), 49-100.

Mobbs, D., Eckert, M. A., Mills, D., Korenberg, J., Bellugi, U., Galaburda, A. M., et al. (2007). Frontostriatal dysfunction during response inhibition in Williams syndrome. Biological Psychiatry, 62(3), 256-261.

Polderman, T. J. C., de Geus, E. J. C., Hoekstra, R. A., Bartels, M., van Leeuwen, M., Verhulst, F. C., et al. (2009). Attention problems, inhibitory control, and intelligence index overlapping genetic factors: A study in 9-, 12-, and 18-year-old twins. Neuropsychology, 23(3), 381.

Porter, M. A., Coltheart, M., \& Langdon, R. (2007). The neuropsychological basis of hypersociability in Williams and Down syndrome. Neuropsychologia, 45(12), 2839-2849.

Rabbitt, P., Lowe, C., \& Shilling, V. (2001). Frontal tests and models for cognitive ageing. European Journal of Cognitive Psychology, 13(1-2), 5-28.

Raitan, R. (1958). Validity of the Trail Making Test as an indicator of organic brain damage. Perceptual and Motor Skills, 8(3), $271-276$.

Rhodes, S. M., Riby, D. M., Park, J., Fraser, E., \& Campbell, L. E. (2010). Executive neuropsychological functioning in individuals with Williams syndrome. Neuropsychologia, 48(5), 1216-1226.

Rockstroh, S., \& Schweizer, K. (2001). The contributions of memory and attention processes to cognitive abilities. The Journal of General Psychology, $128(1)$, $30-42$.

Sampaio, A., Férnandez, M., Henriques, M., Carracedo, Á., Sousa, N., \& Gonçalves, Ó. F. (2009). Cognitive functioning in Williams Syndrome: A study in Portuguese and Spanish patients. European Journal of Paediatric Neurology, 13(4), 337-342.

Searcy, Y. M., Lincoln, A. J., Rose, F. E., Klima, E. S., Bavar, N., \& Korenberg, J. R. (2004). The relationship between age and IQ in adults with Williams syndrome. Journal Information 109(3).

Semel, E. M., \& Rosner, S. R. (2003). Understanding Williams syndrome: Behavioral patterns and interventions. Lawrence Erlbaum.

Spreen, O., \& Strauss, E. (1991). A compendium of neuropsychological tests: Administration, norms, and commentary. New York: Oxford Univ Press Inc.

Strømme, P., Bjømstad, P. G., \& Ramstad, K. (2002). Prevalence estimation of Williams syndrome. Journal of Child Neurology, $17(4), 269$.

Stroop, J. R. (1935). Studies of inference in serial verbal reactions. Journal of Experimental Psychology, 18, 643-662.

Udwin, O., Davies, M., \& Hosylin, P. (1996). A longitudinal study of cognitive abilities and educational attainment in Williams syndrome. Developmental Medicine and Child Neurology, 38(11), 1020-1029.

Wechsler, D. (1997). Wechsler adult intelligence scale. Manual (3rd ed.). San Antonio: Psychological Corporation.

Weschler, D. (2003). Weschler intelligence scale for children(4th ed.). 